\title{
A review of the pharmacogenomics of buprenorphine for the treatment of opioid use disorder
}

\author{
Hemanuel Arroyo Seguí', Kyle Melin², Darlene Santiago Quiñones ${ }^{3}$, Jorge Duconge ${ }^{3}$ \\ 'School of Pharmacy, University of Maryland, Baltimore, MD 21201, USA. \\ ²Department of Pharmacy Practice, School of Pharmacy, University of Puerto Rico Medical Sciences Campus, San Juan, PR \\ 00936, USA. \\ ${ }^{3}$ Department of Pharmaceutical Sciences, School of Pharmacy, University of Puerto Rico Medical Sciences Campus, San Juan, \\ PR 00936, USA.
}

Correspondence to: Dr. Kyle Melin, Department of Pharmacy Practice, School of Pharmacy, University of Puerto Rico Medical Sciences Campus, PO BOX 365067, San Juan, PR 00936, USA.E-mail: kyle.melin@upr.edu

How to cite this article: Arroyo Seguí H, Melin K, Santiago Quiñones D, Duconge J. A review of the pharmacogenomics of buprenorphine for the treatment of opioid use disorder. J Trans/ Genet Genom 2020;4:263-77.

http://dx.doi.org/10.20517/jtgg.2020.35

Received: 31 May 2020 First Decision: 29 Jun 2020 Revised: 1 Jul 2020 Accepted: 10 Jul 2020 Available online: 30 Jul 2020

Academic Editor: Ramón Cacabelos Copy Editor: Cai-Hong Wang Production Editor: Jing Yu

\begin{abstract}
As the opioid epidemic continues to grow across the United States, the number of patients requiring treatment for opioid use disorder continues to climb. Although medication-assisted treatment presents a highly effective tool that can help address this epidemic, its use has been limited. Nonetheless, with easier dosing protocols (compared to the more complex dosing required of methadone due to its long and variable half-life) and fewer prescribing limitations (may be prescribed outside the setting of federally approved clinics), the increase in buprenorphine use in the United States has been dramatic in recent years. Despite buprenorphine's demonstrated efficacy, patient-specific factors can alter the response to the medications, which may lead to treatment failure in some patients. Clinical characteristics (sex, concurrent medications, and mental health comorbidities) as well as social determinants of health (housing status, involvement with the criminal justice system, and socioeconomic status) may impact treatment outcomes. Furthermore, a growing body of data suggests that genetic variations can alter pharmacological effects and influence therapeutic response. This review will cover the available pharmacogenomic data for the use of buprenorphine in the management of opioid use disorders. Pharmacogenomic determinants that affect opioid receptors, the dopaminergic system, metabolism of buprenorphine, and adverse events are discussed. Although much of the existing data comes from observational studies, clinical research is ongoing.
\end{abstract}

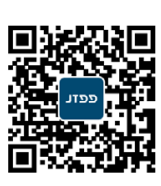


Nevertheless, the development of pharmacogenomic-guided strategies has the potential to reduce opioid misuse, improve clinical outcomes, and save healthcare resources.

Keywords: Pharmacogenomics, pharmacogenetics, buprenorphine, opioid use disorder, opioids, personalized medicine, medication-assisted treatment, buprenorphine/naloxone

\section{INTRODUCTION}

The United States (US) has seen a surge in prescription and illicit use of opioids in the last twenty years, creating an unprecedented healthcare and socioeconomic crisis. Approximately 2 million people in the US have opioid use disorders (OUD) resulting from prescription usage alone ${ }^{[1]}$. This life-threatening chronic brain disease is associated with a significant increase in early death, resulting from overdose, trauma, suicide or infectious diseases (e.g., Hepatitis C, HIV/AIDS) ${ }^{[2]}$. From 1999 to 2018, approximately 450,000 people died in the US due to opioid overdoses ${ }^{[3]}$. Healthcare costs, loss of productivity, and criminal involvement creates substantial socioeconomic burden ${ }^{[4]}$. The Centers for Disease Control and Prevention estimate costs of more than $\$ 78$ billion per year in the US from prescription opioid misuse, and others have estimated an additional loss of $\$ 50$ billion from heroin use $\mathrm{e}^{[5,6]}$.

The prolonged and repeated administration of opioids over time causes lasting effects on neuronal structure and function ${ }^{[4]}$. The 5 th edition of the Diagnostic and Statistical Manual of Mental Disorders defines OUD as a problematic pattern of misuse resulting in clinically significant impairment or distress ${ }^{[7]}$. OUD can be effectively managed using medication-assisted treatment (MAT), a combination of pharmacological and behavior-based interventions such as, cognitive-behavioral therapy, contingency plans, 12-step programs and support groups ${ }^{[2]}$. Evidence strongly recommends the use of medications in all patient groups, including adolescents, pregnant women and older adults ${ }^{[4,8-14]}$. Pharmacotherapy can reduce morbidity and mortality by restoring brain function, supporting opioid abstinence and reducing risk for overdose death ${ }^{[4,15,16]}$. OUD treatment can also lower the risk of blood-borne infections and help patients recover social functionality, easing reintegration into their communities ${ }^{[17-19]}$.

After initial detoxification (i.e., opioid withdrawal management), patients should be introduced to maintenance therapy with MAT. FDA-approved medications for maintenance therapy include methadone, buprenorphine and extended-release naltrexone $e^{[2,4]}$. All three medications target receptors within the opioid system and work by reducing opioid cravings and/or decreasing the response to future drug use $e^{[4]}$. Due to their direct agonistic effect on opioid $\mu$-receptors, methadone and buprenorphine are also indicated for symptomatic relief of acute opioid withdrawal ${ }^{[2]}$. Despite the availability of these life-saving medications, most patients do not receive adequate treatment ${ }^{[4]}$. Social barriers and policy regulations limit the number of patients who receive pharmacotherapy, especially in underserved communities (e.g., African Americans, Hispanics). Methadone can only be dispensed in federally approved clinics [i.e., opioid treatment programs (OTPs)], restricting access for many patients ${ }^{[2]}$. Buprenorphine can be prescribed in office-based settings, but providers require completion of an 8 -hour training course, and obtain a waiver from the Drug Enforcement Administration ${ }^{[20]}$. About $2 \%-3 \%$ of providers in the US have such waivers ${ }^{[4,21]}$.

Furthermore, patient-specific factors can alter the response to these medications, which may lead to treatment failure in some patients ${ }^{[4,22,23]}$. Clinical characteristics such as sex, concurrent medications, and mental health comorbidities have all been shown to affect patient variability in response to pharmacotherapy ${ }^{[24-26]}$. Social determinants of health such as housing status, involvement with the criminal justice system, and socioeconomic status may also impact treatment outcomes ${ }^{[4,27,28]}$. Lastly, genetic variations can alter pharmacological effects and influence therapeutic response ${ }^{[29-31]}$. Pharmacogenomic 
studies evaluate genotypic-phenotypic associations and their potential impact on pharmacokinetic (e.g., metabolic function, dosing requirements) and pharmacodynamic (e.g., receptor activity, treatment efficacy) parameters. In recent years, pharmacogenomic research for OUD has accelerated.

To date, the majority of pharmacogenomic studies for OUD treatment have focused on methadone use, where a number of relevant pharmacogenes have been identified. Methadone is a full agonist at the $\mu$-opioid receptor, with antagonistic activity at the $\mathrm{N}$-methyl-D-aspartate (NMDA) receptor ${ }^{[20]}$. This NMDA antagonism can potentially cause cardiac toxicity, especially in patients with an elevated QTc interval. Regarding pharmacogenes, $\mathrm{CYP} 2 \mathrm{~B} 6$ variants (e.g., ${ }^{*} 4,{ }^{*} 6,{ }^{*}$ alleles) have been associated with alterations in metabolic rate and plasma methadone concentrations, which could determine dosing requirements ${ }^{[32-34]}$. OPRD1 variants (e.g., rs678849) have been shown to impact treatment outcomes in African American patients, while OPRM1 variants (e.g., rs10485058, rs3192723) may impact efficacy in European and Asian patients ${ }^{[31,35]}$. UGT2B7 variants (e.g., rs6600879, rs4554144) may also determine severity of opioid withdrawal in some populations ${ }^{[36]}$. The evidence related to the pharmacogenomics of methadone has recently been reviewed elsewhere and, therefore, will not be further discussed here ${ }^{[37,38]}$.

Buprenorphine is a high-affinity, $\mu$-receptor partial agonist ${ }^{[20]}$. It also possesses antagonistic activity on $\kappa$-receptors, and weak agonist activity on $\delta 1$-receptors. Partial agonism leads to effective analgesic effects and abatement of withdrawal symptoms while reducing the risk of respiratory depression observed with methadone and other opioids ${ }^{[20]}$. Additionally, due to its effects on $\kappa$-receptors, clinical data have suggested that buprenorphine may possess antidepressant benefits, potentially representing a better option for patients with comorbid depression ${ }^{[39,40]}$. Buprenorphine has also been co-formulated with naloxone as a sublingual film or tablet to discourage intravenous administration, which results in the immediate onset of euphoric effects ${ }^{[20]}$. Naloxone is a high-affinity $\mu$-receptor antagonist that displaces other opioid ligands, essentially blocking and reducing their pharmacological effects (e.g., euphoria, respiratory depression). With easier dosing protocols (compared to the more complex dosing required of methadone due to its long and variable half-life) and fewer prescribing limitations (may be prescribed outside the setting of federally approved clinics), the increase in buprenorphine use has been dramatic in recent years ${ }^{[21]}$. The number of patients receiving therapy from non-OTP facilities increased from 1,670 in 2004 to 54,488 in 2015, an increase of three thousand percent. However, pharmacogenomic studies including buprenorphine have lagged behind those of methadone. Understanding how to tailor drug treatment in OUD is essential to reducing opioid misuse, improving treatment adherence, and saving healthcare resources. This review will cover the available pharmacogenomic data for the use of buprenorphine in the management of OUDs.

\section{PHARMACOGENES: OPIOID RECEPTORS}

Activity on all three G-protein coupled receptors of the opioid receptor family ( $\mu, \delta$ and $\kappa$ ), differentiates buprenorphine from other opioid-based medications ${ }^{[41]}$. These receptors are structurally and functionally related, but exhibit variations in ligand affinity and cellular distribution ${ }^{[42]}$. The main effects of opioids (i.e., analgesia, reward, adverse effects) are mediated through $\mu$-receptor binding, while $\delta$ - and $\kappa$-receptors help modulate these effects via different biological mechanisms. Alterations in opioid receptor expression and function (i.e., genetics), may impact substance dependence risk and influence treatment response ${ }^{[31,42]}$.

\section{$\mu$-Opioid receptor gene (OPRM1)}

As the primary target in opioid therapeutics, it is reasonable to assume that activity on $\mu$-receptors plays a crucial role in opioid dependence, and that variations in the genes encoding these receptors may impact clinical outcomes. Although OPRM1 variants (e.g., rs9479757G>A) have been associated with heroin dependence risk, pharmacogenomic analyses of OPRM1 variants have demonstrated conflicting results in OUD treatment response ${ }^{[43]}$. 
Data from the randomized clinical trial Starting Treatment With Agonist Replacement Therapies (START), has been used to perform several pharmacogenomic analyses of OUD treatment outcomes ${ }^{[44]}$. Genotypic information was available for $60 \%(n=764 / 1,267)$ of the patient population, which was primarily composed of European Americans $(n=599)$, with few African Americans $(n=79)$ and other ethnicities $(n=96)^{[29]}$. Over a 24-week period, participants treated with methadone $(n=364,66 \%$ male $)$ or buprenorphine/naloxone ( $n=410,71 \%$ males) were submitted to weekly urine drug screens. From the several pharmacogenomic studies conducted from this trial, only two evaluated OPRM1 variants ${ }^{[29,30]}$. No significant results were observed between variants and buprenorphine response in either study. One study compared the genotypes of all participants at the OPRM1 variant rs1799971, with dropout rate and dosing requirements ${ }^{[29]}$. The other used a sample of European Americans $(n=582)$ and analyzed the effects of single-nucleotide polymorphisms (SNPs) in an untranslated region ( $3^{\prime}$ ) of OPRM1, on the number of opioid-positive urine tests ${ }^{[30]}$. Although no differences were found with buprenorphine, this second study did show an association between the OPRM1 variant rs10485058 and methadone-treated patients. AA genotypes at this locus were less likely to have opioid-positive urine tests than carriers of the G-allele ( $R R=$ $0.76, P=0.0064)$.

In addition to OUD trials, one study evaluated the effect of the OPRM1 118A>G (rs1799971) polymorphism on the analgesic effect of buprenorphine ${ }^{[45]}$. The study was conducted in a Spanish population $(n=93,77 \%$ male) with critical limb ischemia who were hospitalized for revascularization and treated with transdermal buprenorphine for pain. No significant association was found between rs1799971 and the analgesic effect of buprenorphine, although significant findings were observed with other pharmacogenomic variables, which will be discussed later in this review. On the other hand, a study evaluating an individualized opioid deprescription program with buprenorphine, did show a significant association between the same OPRM1 variant rs1799971 and morphine equivalent daily dose (MEDD) requirements ${ }^{[46]}$. Participants included patients of European ancestry ( $n=88,64 \%$ female) with chronic non-cancer pain, who were using opioids long-term (> 6 months) and were diagnosed with prescription opioid dependence. Interventions involved a slow-tapering process, with a $30 \%$ reduction in opioid dose and rotation to buprenorphine and/or tramadol. Carriers of the rs1799971 variant required significantly higher MEDD $(P<0.05)$ for analgesic control and prevention of withdrawal symptoms. No associations were found between OPRM1 and program response.

\section{ס-Opioid receptor gene (OPRD1)}

Multiple studies have demonstrated the impact of $\delta$-Opioid receptor gene (OPRD1) genetic variants on substance dependence risk ${ }^{[42,47]}$. As for OUD treatment, a series of SNPs located on intron 1, may be predictive of outcomes in some patient populations receiving buprenorphine. A patient cohort from the START trial was used to evaluate the effects of OPRD1 variants on opioid abstinence, which was measured by urine drug screens ${ }^{[31]}$. This sample was primarily composed of European Americans ( $n=566,68 \%$ male) and some African Americans ( $n=77,69 \%$ male). Although no significant differences were observed in the amount of opioid-positive tests when comparing methadone and buprenorphine, the rs678849 variant of $O P R D 1$ was significantly associated with treatment outcomes in African Americans for both medications. In the buprenorphine group, African Americans with the CC genotype $(n=24)$ were more likely to have opioid-positive drug screens than CT/TT genotypes $(n=17, \mathrm{RR}=2.17, P=0.008)$. The opposite was observed for the methadone group, as African Americans with the CC genotype $(n=21)$ were less likely to have opioid-positive drug screens than carriers of the T-allele $(n=15)(R=0.52, P=0.001)$. No significant results were shown in European Americans for any OPRD1 genetic variant. The researchers concluded that for African Americans, matching rs678849-related genotypes to pharmacotherapy could improve treatment efficacy overall ${ }^{[31]}$. Estimates have indicated that half of all African Americans may possess the CC genotype $^{[37]}$. A follow-up analysis was performed to replicate the results of this study using an independent cohort of African Americans ${ }^{[48]}$. This replication study confirmed that the rs678849 genotypes were related to buprenorphine outcomes: CC genotype was more likely to have opioid-positive tests than CT/TT 
genotypes $(\mathrm{RR}=1.69, P=0.021)$. This analysis was unable to confirm the previously identified association between the $\mathrm{C}$-allele and treatment success in the methadone group. Of note, the methadone group in this cohort was relatively small with only 22 participants.

In addition to the OPRD1 variant rs678849, two other intronic SNPs have been identified as clinically relevant in buprenorphine therapeutics. Using the European American sample from the START trial, a pharmacogenomic analysis was conducted to evaluate sex-specific differences on clinical outcomes ${ }^{[49]}$. $O P R D 1$ variants were compared between males and females undergoing methadone or buprenorphine/ naloxone treatment. No significant interactions were observed for males on either treatment, nor females on methadone. However, in females treated with buprenorphine $(n=81)$, genotypes at rs581111 and rs529520 predicted therapeutic response. During the 24-week treatment period, females with a GG genotype at rs581111 were more likely to have opioid-positive tests than AA/AG genotypes combined (RR $=1.72, P=0.031)$. At rs529520, females with the AA genotype were more likely to have opioid-positive tests than females with the $\mathrm{CC}$ genotype $(\mathrm{RR}=1.65, P=0.025)$. Outcomes in carriers of the AC genotype were not significantly different from those with the AA genotype. The researchers concluded that genotypes at these two OPRD1 variants may be useful when considering OUD pharmacotherapy for females, but further validation is warranted. Interestingly, sex and gender differences have previously been shown to impact OUD outcomes, as well as buprenorphine pharmacokinetic and pharmacodynamic parameters ${ }^{[4,24,50-52]}$.

\section{K-Opioid receptor gene (OPRK1)}

$\kappa$-Opioid receptors may play an important role in buprenorphine therapeutics, especially for patients with comorbid depression. The antidepressant effects displayed by buprenorphine are related to its antagonistic activity at these receptors ${ }^{[39,40]}$. Uncontrolled psychiatric illnesses can negatively impact OUD treatment outcomes, and thus, a medication that could potentially provide benefits for both disorders would be clinically advantageous ${ }^{[53,54]}$. To date, one study has evaluated OPRK1 genetic variants on buprenorphine response ${ }^{[55]}$. The patient population was composed of Western Europeans ( $n=107,81 \%$ male) who had a history of heroin-dependence for 4-7 years. Buprenorphine was administered for a 6-month period, and participants were classified as responders or non-responders on the basis of their clinical outcomes (e.g., relapse, treatment completion). Genotypes at the OPRK1 36G>T SNP (rs1051660) were compared between response groups, but no significant differences were observed. Of note, due to the low numbers of TT and GT genotypes observed in the study population, the two genotypes were collapsed, and the comparison was made between GG versus GT/TT (i.e., dominant genetic model). In addition, the small number of patients affected by mental health comorbidities precluded an analysis of the association between gene variants and psychiatric illnesses. Despite the negative finding of this small study, further investigations of this variant in other populations and of other genetic variants influencing the function of the $\kappa$-opioid system, are needed to assess the effects of OPRK1 genetic variants on OUD treatment outcomes. Of note, this study by Gerra et al ${ }^{[55]}$ did find a significant association with a dopamine-related pharmacogene, which will be discussed later.

\section{Discussion}

Preliminary evidence suggests that some opioid receptor genetic variants may be associated with buprenorphine treatment outcomes in OUD. The strongest evidence to date shows an association with variants in intronic OPRD1 SNPs and treatment efficacy, but race and sex may modulate these effects. The rs678849 variant in particular shows promise as a potential clinical variable that could be useful to direct individuals toward buprenorphine (carriers of CT/TT genotypes) or methadone (carriers of CC genotype) in African Americans. Additionally, the rs581111 and rs529520 variants have the potential for utility among females of European ancestry, especially given the differences observed in the opioid system between sexes. Nonetheless, further research is needed with larger and more diverse populations to better understand the role of these variants and other opioid receptor variants in pharmacogenomics-guided treatments. 


\section{PHARMACOGENES: DOPAMINERGIC SYSTEM}

As discussed, buprenorphine acts on $\kappa$-receptors via antagonism. These receptors are expressed in dopaminergic neurons, where they modulate the release of dopamine ${ }^{[40]}$. The dopaminergic system has been tied to signaling pathways related to reward, mood, and behavior ${ }^{[56]} \cdot \kappa$-Receptor agonists inhibit the release of dopamine, which can induce stress and dysphoria ${ }^{[57]}$. Furthermore, substance-dependent states are associated with $\kappa$-opioid system overdrive, which may reinforce drug-seeking behaviors due to the high levels of stress. Buprenorphine administration may antagonize these receptors, helping to normalize levels of dopamine in the nucleus accumbens, and potentially improving mood and impulsive behavior tendencies. Other monoamines (e.g., serotonin) may also be involved in these antidepressant effects. To date, several studies have evaluated the relationship between dopaminergic pharmacogenes and the clinical outcomes of buprenorphine therapy.

D2-receptor gene (DRD2) dysfunction has been related to drug-seeking behavior, and therefore, genetic variants may influence OUD treatment response ${ }^{[58]}$. Although the DRD2 TaqI A1 polymorphism has been associated with poor outcomes among methadone patients, no such association has yet been observed with buprenorphine ${ }^{[59]}$. A retrospective study evaluated the presence of the DRD2 TaqI A1 allele in treatment response with methadone ( $n=46,57 \%$ males) and buprenorphine $(n=25,68 \% \text { females })^{[60]}$. The patient population was mostly composed of Australians with European ancestry (88\%). No significant associations were found related to buprenorphine dose or response, although methadone patients who were DRD2 TaqI A1 carriers experienced less withdrawal symptoms than non-carriers $(P=0.04)$.

The previously mentioned study by Gerra et al ${ }^{[55]}$ also evaluated the presence of different alleles at the dopamine transporter gene (SLC6A3/DAT1) on buprenorphine response. As mentioned, participants ( $n$ $=107,81 \%$ males) were classified as responders or non-responders. Non-responders showed continuous use of heroin, severe psychiatric distress, medication diversion and/or dropout of treatment. A significant difference was observed between groups, as all carriers of the 11-repeat allele were able to complete treatment for the duration of the study, without significant disturbances $(P=0.001)$. In addition, the frequency of the 10-repeat allele was higher in non-responders ( $65 \%$ vs. 56\%), but statistical significance was not established.

\section{Discussion}

The research that exists to date, regarding the role of dopaminergic pharmacogenes on buprenorphine therapeutics is still limited. The study of the DRD2 TaqI A1 allele by Barratt et al. ${ }^{[60]}$ was likely underpowered to identify meaningful associations, particularly in the buprenorphine arm. However, their preliminary findings in the methadone group suggest further research is justified. The results from Gerra et al. ${ }^{[5]}$ suggest a potential clinical utility in SLC6A3/DAT1 variants that also warrants further study.

\section{PHARMACOGENES: METABOLISM}

The metabolic rate of drugs can vary between individuals, making dosing adjustments necessary to achieve the desired effects. Buprenorphine is extensively metabolized in the liver via N-dealkylation by cytochrome $\mathrm{P} 450$ (CYP450) enzymes, producing nor-buprenorphine, the major active metabolite ${ }^{[61,62]}$. To a lesser extent, both buprenorphine and nor-buprenorphine undergo glucuronidation by UGT [Figure 1]. CYP3A4 may be considered the primary CYP450 enzyme in the metabolism of buprenorphine, but other enzymes involved have been identified. Using human liver microsomes, a study found that CYP3A4 and CYP2C8 were able to produce the active metabolite in considerable concentrations ${ }^{[61]}$. CYP $3 \mathrm{~A} 5$ and CYP $3 \mathrm{~A} 7$ were also identified as contributors in the metabolic pathway. Approximately $90 \%$ of buprenorphine was shown to be metabolized by CYP3A enzymes, and thus, genetic variations due to ancestry and/or sex differences in these enzymes may impact buprenorphine exposure ${ }^{[63]}$. In addition to the CYP450 system, the UGT2B7 
norbuprenorphine

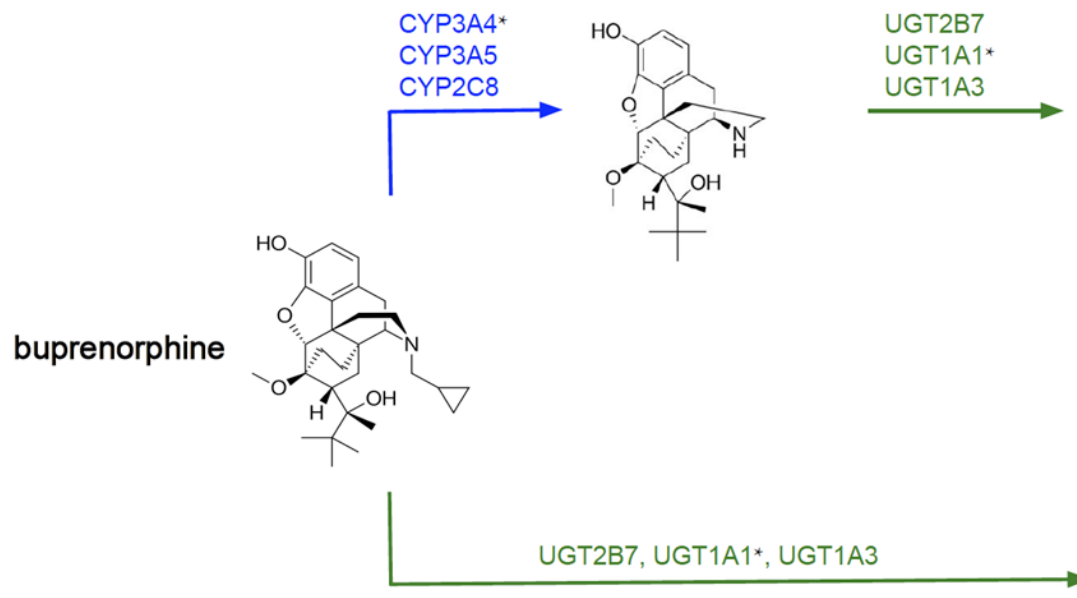

norbuprenorphine glucuronide

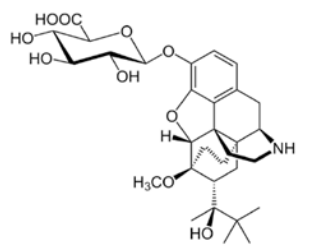

buprenorphine glucuronide

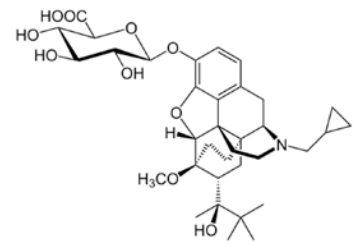

Figure 1. The metabolic pathways of buprenorphine. *Variants of this gene have been found to be clinically relevant

gene has been identified as playing a minor role in buprenorphine metabolism ${ }^{[64]}$. In a study with human liver microsomes, the presence of the $U G T 2 B 7$ promoter (G-842A) mutation resulted in higher buprenorphine glucuronidation $\mathrm{V}_{\max }(80 \%$ on average) and a higher glucuronidation rate in non-carriers (but not in carriers) of the UGT1 $\mathrm{Al}^{\star} 28$ allele $(P=0.0352)$.

Phenotypic classifications have been developed to categorize functional variants of CYP3A4, as poor (PM), intermediate (IM), extensive (EM), or ultrarapid (UM) metabolizers ${ }^{[65]}$. Without dose adjustments, poor metabolizers may have higher than normal plasma levels of buprenorphine, potentially increasing the risk for adverse events. Similarly, ultrarapid metabolizers may have lower plasma levels, which may induce opioid cravings and/or withdrawal symptoms. This particular scenario was observed in a case report involving an African American male undergoing OUD management with buprenorphine/naloxone ${ }^{[65]}$. During every medical appointment, a urine screening was performed to assess adherence and detect the use of unauthorized or illicit substances. This patient had no significant socioeconomic barriers (i.e., married, stable home and employed) and was being treated with a buprenorphine daily dose of $28 \mathrm{mg}$. For a few years the patient was adherent with no use of other opioids, although occasionally used synthetic cannabinoids. However, following a dosage decrease to $24 \mathrm{mg}$ due to a change in insurance coverage, the patient reported withdrawal symptoms and had multiple recurrences with morphine, methadone, benzodiazepines and synthetic cannabinoids. Pharmacogenomic testing found the patient to have a CYP $3 A 4^{*} 1{ }^{*} 1 \mathrm{~B}$ diplotype, which has been associated with an increased metabolic rate ${ }^{[6,67]}$. Less than a year later, the third-party payer dose-limiting policy was cancelled and the patient went back to $28 \mathrm{mg}$ for one month, and then increased to $32 \mathrm{mg}$. During the next 4 months with $32 \mathrm{mg}$, the patient did not use any unauthorized substances. As a $C Y P 3 A 4^{*} 1 \mathrm{~B}$ carrier, the patient was classified as an ultrarapid metabolizer, consistent with a higher dose requirement.

The same research group then conducted a retrospective cohort study to evaluate $C Y P_{3} A 4$ and $C Y P_{3} A 5$ polymorphisms on buprenorphine response (i.e., dosing, withdrawal and relapse) ${ }^{[67]}$. The patient population was mostly composed of African Americans ( $n=111 / 113,76 \%$ males). Participants were classified by their CYP3A4 phenotype, and the majority of the patient population were ultrarapid metabolizers $(82 \%)$, carrying at least one ${ }^{\star} 1 \mathrm{~B}$ allele. Clinical outcomes assessed included withdrawal instances, use of 
unauthorized substances (i.e., urine drug screens), and dosing comparisons between standard-of-care (SOC) and PGx guided treatment. With PGx-guided dosing (that allowed for higher dosing), carriers of the ${ }^{*} 1 \mathrm{~B}$ allele (homozygous or heterozygous) had significantly less withdrawal symptoms compared to patients on SOC dosing $(P=0.0294)$. No significant differences were observed with CYP3A5 genetic variants or the use of unauthorized substances. The researchers concluded that $C Y \mathrm{P}_{3} A 4$ genetic variants can impact clinical outcomes, and therefore, PGx-guided dosing should be implemented in buprenorphine therapeutics, especially for African American patients.

Using a sample from the previously mentioned START trial $(n=764 / 1,267)$ composed of European Americans $(n=599)$, African Americans $(n=79)$, and other ethnicities $(n=96)$, a different study evaluated variants in six pharmacokinetic genes (i.e., CYP1A2, CYP2B6, CYP2C19, CYP2C9, CYP2D6 and CYP3A4) on dropout rate and mean dose ${ }^{[29]}$. No significant associations were identified for any variants of these genes in either the buprenorphine or methadone treatment arm.

Blanco et al. ${ }^{[45]}$ examined the effects of both the UGT2B7 802C>T (rs7439366) polymorphism and the CYP3A4 290A $>\mathrm{G}$ (rs2740574) polymorphism on analgesic response to buprenorphine. As previously mentioned, the study included 93 patients with critical limb ischemia who were treated with transdermal buprenorphine for pain. In this study, patients who were AA homozygotes for the CYP3A4 gene showed the best response to analgesic treatment $(P=0.003)$, but no association was identified between the UGT2B7 polymorphism and analgesic response to buprenorphine.

\section{Discussion}

Buprenorphine dose adjustments based on CYP3A4 functional status are the only PGx-guided interventions that have been implemented and evaluated to date. Findings from Ettienne et al. ${ }^{[65,67]}$ suggest that dosing patients by $\mathrm{CYP}_{3} \mathrm{~A} 4$ phenotype can improve treatment outcomes. Patients with an increased metabolic rate (i.e., EM and UM) may need higher doses to prevent withdrawal symptoms and maintain opioid abstinence. However, to date, these improvements have only been observed in African Americans. The sample from the START trial was mostly composed of European Americans $(n=599 / 764)$ and did not show significant associations between any metabolic gene variant and mean dose or dropout rate. Of note, results did trend towards a potential association between CYP3A4 status and dropout rate, when the buprenorphine and methadone groups were combined. The authors hypothesized that a possible reason for the lack of significance was the low frequency of patients with PM or IM phenotypes in their study population with Western European ancestry. Again, further research with larger and more diverse patient populations is needed to assess the clinical benefits of CYP3A4-guided dosing (or other metabolic genes) for buprenorphine OUD treatment.

\section{PHARMACOGENES: ADVERSE EFFECTS}

Genetic variations can not only influence treatment efficacy, dosing requirements and withdrawal incidence but adverse effects as well. Multiple studies involving methadone-treated patients have found associations between the rate and intensity of adverse effects with several pharmacogenes ${ }^{[38]}$. However, only one study has included buprenorphine in their evaluation of genetic variants and adverse effects. A prospective study analyzed variants in OPRM1, OPRD1, COMT, ARRB2 and $A B C B 1$ on patients participating in an opioid deprescription program with buprenorphine patches ${ }^{[68]}$. The patient population was composed of Europeans with non-cancer chronic pain ( $n=88,64 \%$ females), who had been taking prescription opioids for more than 6 months, and were diagnosed with OUD at the start of the study. AA genotype at the OPRM1 variant s1799971 was associated with a higher incidence of nausea $(P=0.034)$ and gastrointestinal adverse events $(P$ $=0.031)$ when compared with AG/GG genotypes combined. Patients with the CT genotype at the OPRD1 variant rs2234918 experienced less sexual dysfunction than TT/CC genotypes combined $(P=0.001)$. At the COMT variant rs4680, the AG genotype was associated with a lower incidence of loss of libido $(P=0.003)$ 
and skin redness $(P=0.003)$, while the AA genotype was associated with a higher incidence of vomiting $(P$ $=0.003)$. Patients with the TT genotype at the ARRB2 variant rs1045280 were less likely to experience loss of libido $(P=0.021)$ and dry skin $(P=0.024)$ than CC/CT genotypes. No significant differences were found with $A B C B 1$ variants.

\section{Discussion}

To date, the study by Muriel et al ${ }^{[68]}$ provides the only evidence on pharmacogenomic determinants of adverse events for patients with OUD treated with buprenorphine. Several factors, however, make interpretation of the study difficult. One key limitation in understanding the specific relation to buprenorphine is the inclusion of other opioids. The deprescription process included opioid tapering with the addition of tramadol, plus some patients were transitioned to fentanyl patches instead of buprenorphine (the authors do not report what percentage of patients were treated with buprenorphine patches compared to fentanyl patches). In addition, there were conflicting results between genotypes and the frequency of adverse events, when comparing baseline and final visits. For example, the AG genotype at COMT variant rs4680 was associated with less skin redness at baseline, but significantly more (than AA/GG genotypes) at the end of the program $(P=0.007)$. In summary, individual genotypes have the potential to contribute toward the rate and intensity of adverse events in OUD patients treated with buprenorphine. However, more research is needed to better understand the influence of genetic variants on adverse effects.

\section{CONCLUSION}

The prevalence of OUD has reached unparalleled numbers in the last decades, increasing the rates of overdose fatalities and socioeconomic burden. Most of these patients do not receive proper treatment ${ }^{[4]}$, and furthermore, the lack of guidelines for individualized pharmacotherapy may limit the therapeutic benefits of medication-assisted treatment. Patient-specific factors can influence treatment response, but to date, clinicians are not equipped to use this data for therapy optimization. Understanding the pharmacogenomic variables that predict reductions in illicit opioid use, treatment adherence, and incidence of withdrawal symptoms would enhance clinical decisions.

For many years, methadone has been the primary option for medication-assisted treatment for OUD. However, buprenorphine may present a safer and more effective alternative for some patients based on comorbidities (e.g., mental health disorders) and genetic profile. Even though pharmacogenomic studies have accelerated in recent years, research involving PGx-guided strategies for OUD treatment has been limited, especially with buprenorphine. Nonetheless, the available data suggests significant relationships between some pharmacogenes and buprenorphine response [Table 1] ${ }^{[69]}$. Evidence is strongest for variants in opioid receptor pharmacogenes (OPRD1) and metabolic pharmacogenes (CYP3A4), but still limited with regards to dopaminergic pharmacogenes. Of particular clinical value might be genetic markers with ability to potentially direct treatment by identifying patients who may respond favorably to buprenorphine, but poorly to methadone and vice versa.

In conclusion, further research is needed to better understand how pharmacogenomic factors may be proactively used to improve treatment outcomes. As the vast majority of data that currently exists regarding pharmacogenomic determinants of buprenorphine response has been observational, the benefits of incorporating these factors into clinical treatment decisions remain theoretical. Thus, to date, there are no FDA-approved or Clinical Pharmacogenetics Implementation Consortium (CPIC ${ }^{\bullet}$ ) recommendations for directing OUD therapy on the basis of pharmacogenomic testing. Hopefully, prospective clinical trials will better elucidate the role of PGx-guided treatment strategies. Additional research opportunities also exist in larger genome-wide association studies to identify novel genetic variants, investigating other potentially relevant candidate pharmacogenes (e.g., perhaps those affecting the ORL-1/NOP receptors), and research to better understand how other clinical factors such as treatment adherence interact with and mediate 


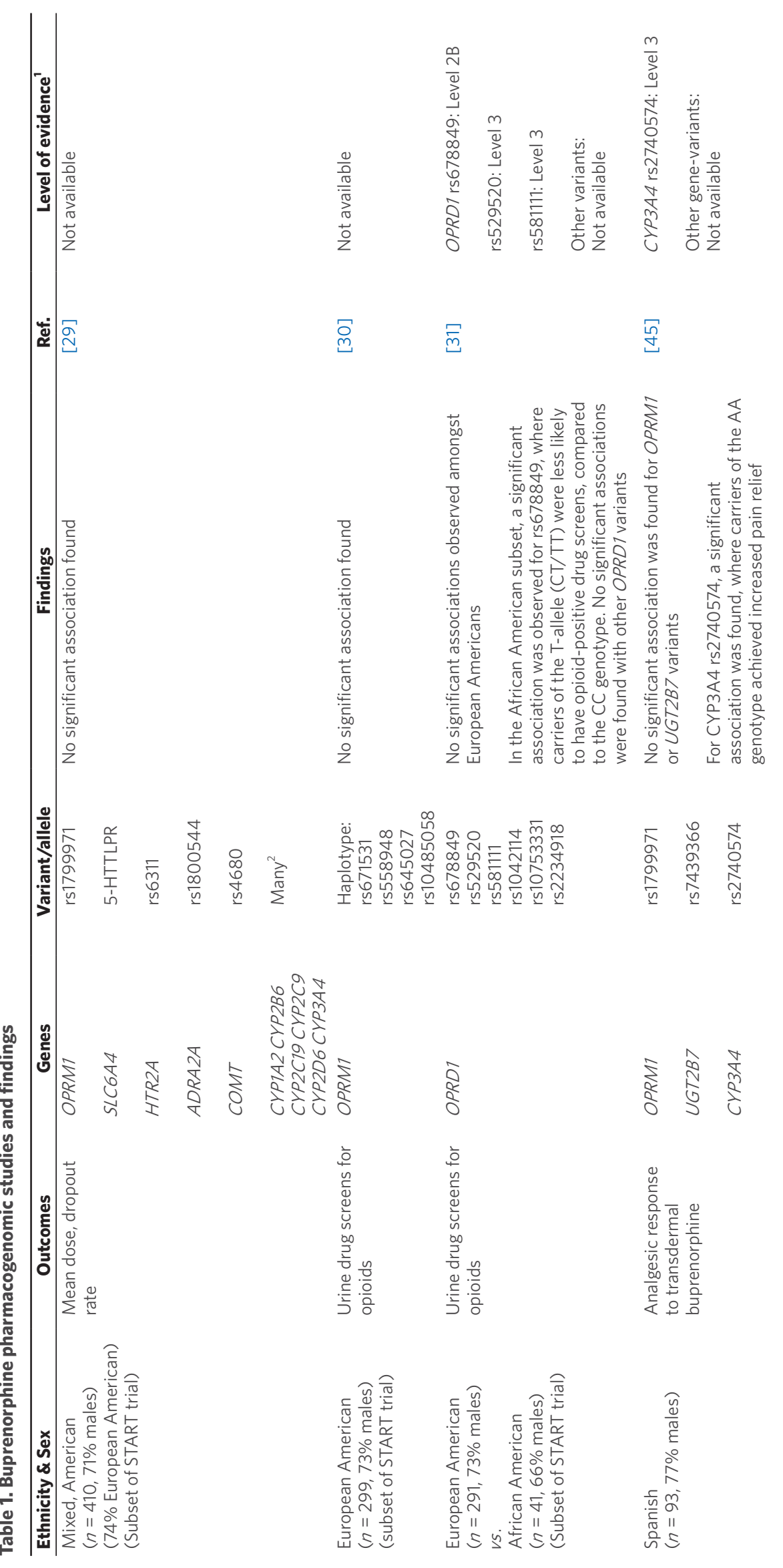




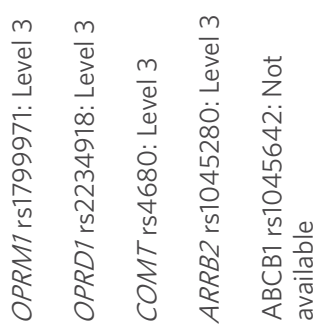

$\infty$
$\substack{0 \\ 0}$

守

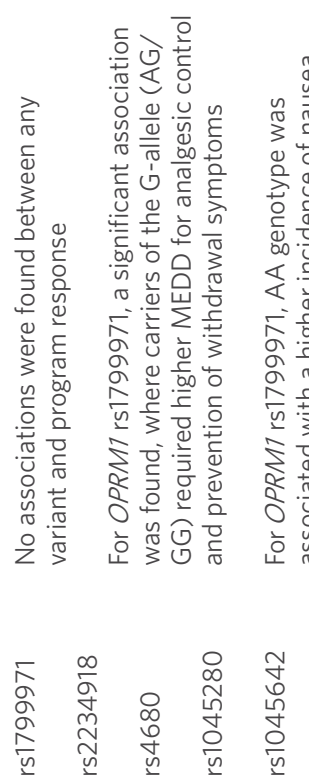

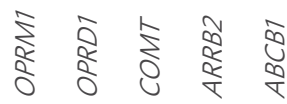

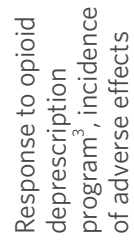

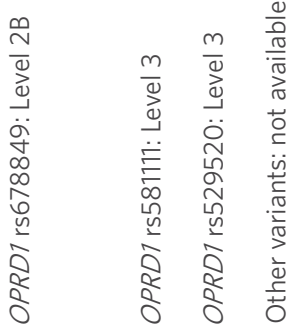

$\stackrel{\infty}{\Xi} \quad \stackrel{g}{巳}$
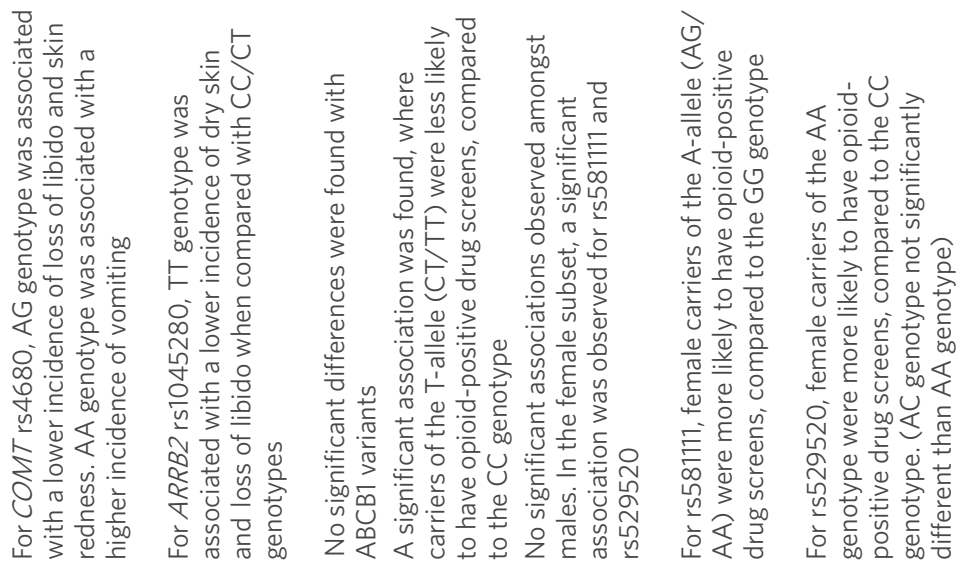

$\begin{array}{ll}\bar{a} & \bar{a} \\ \vdots & 0\end{array}$

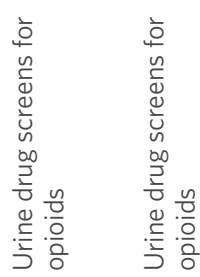

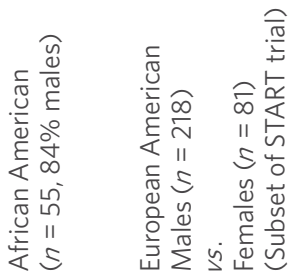



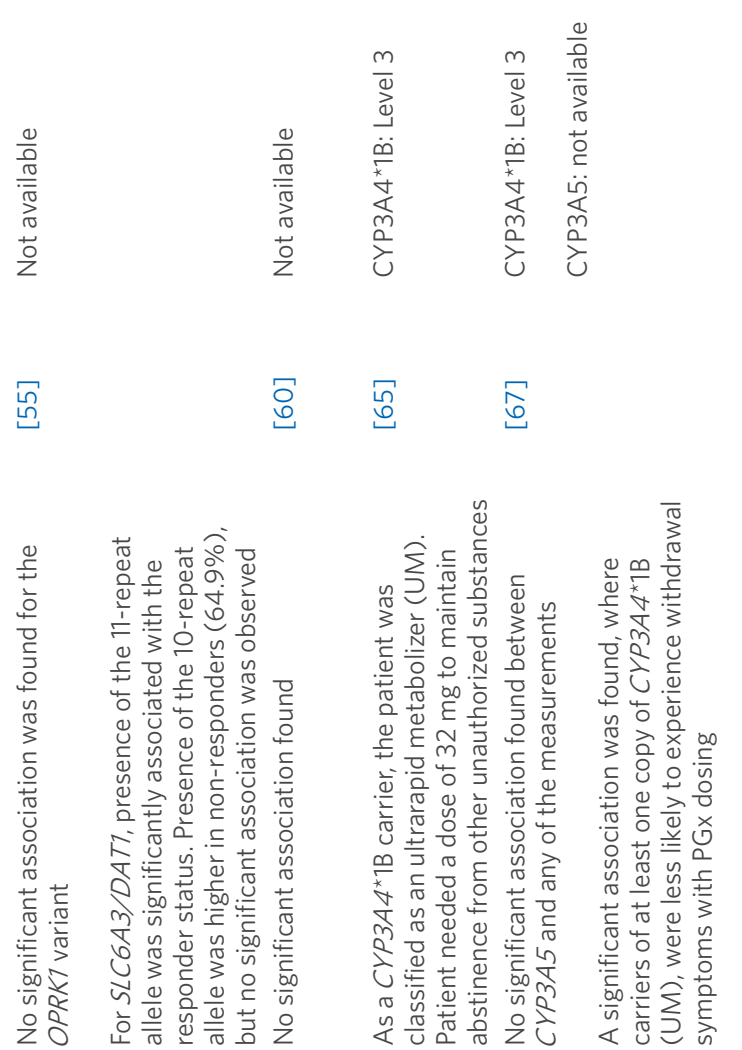

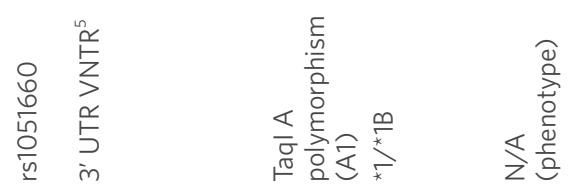

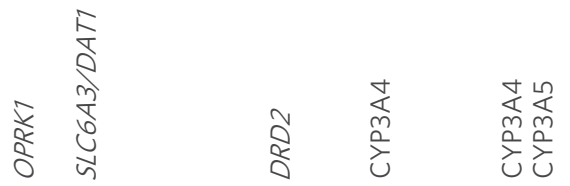

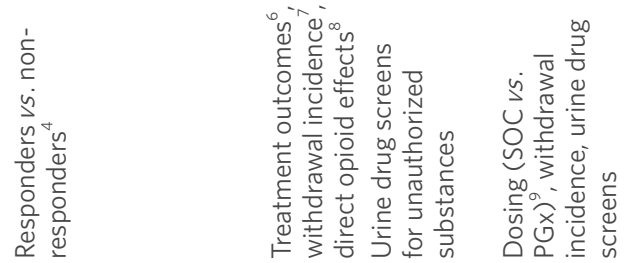

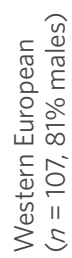

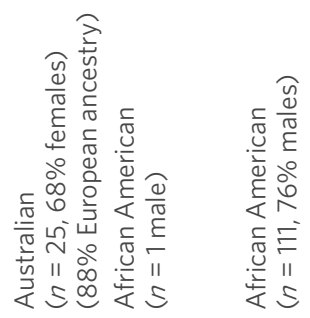

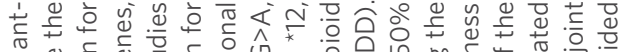

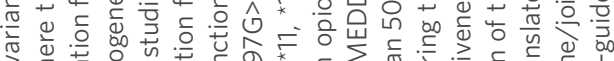

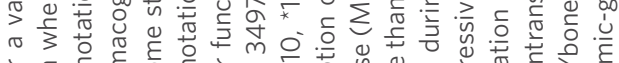

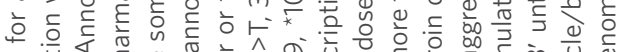

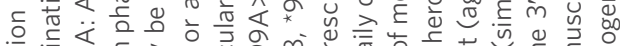

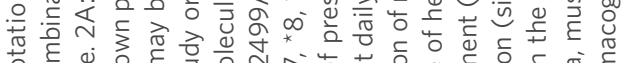

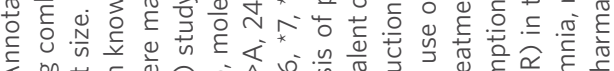

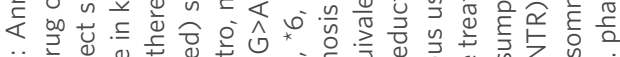

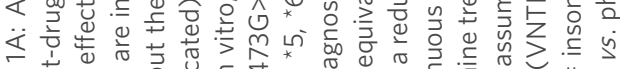

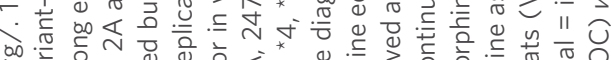

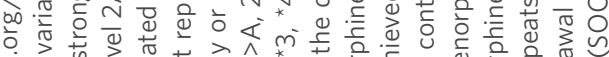

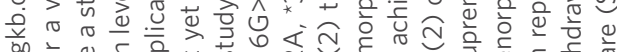

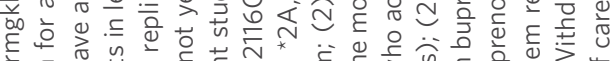

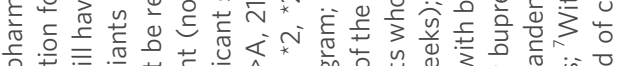

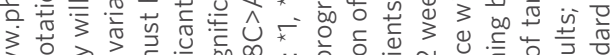

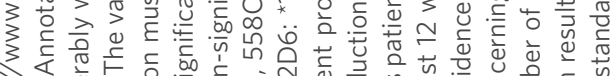

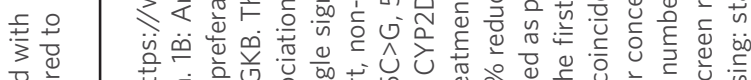

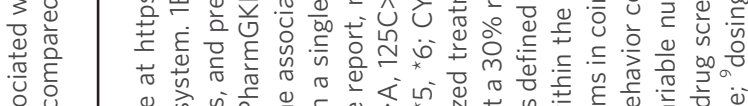

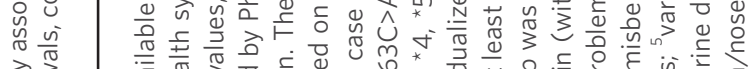

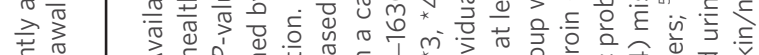

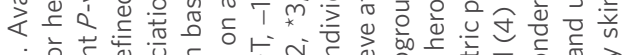

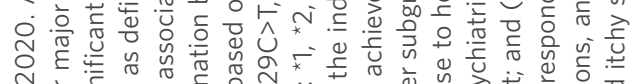

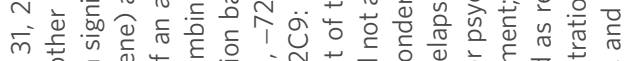

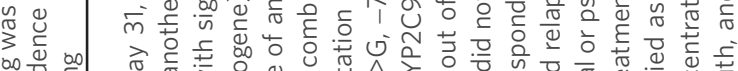
o.

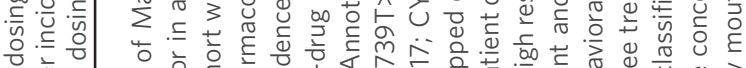

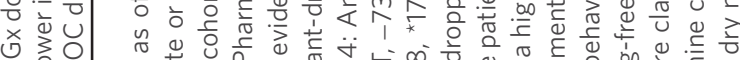

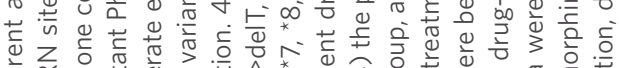

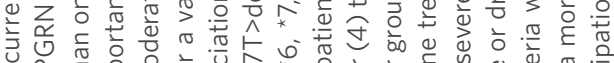

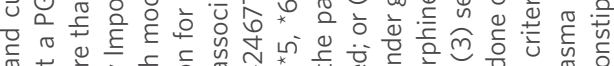

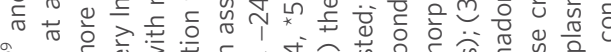

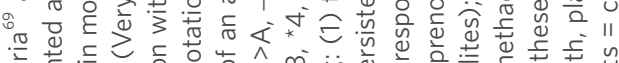

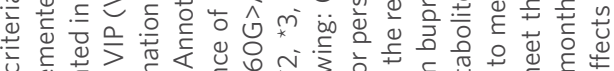

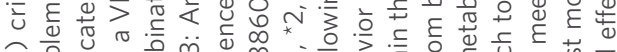

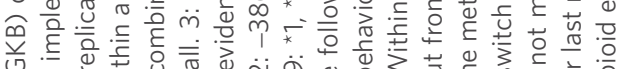

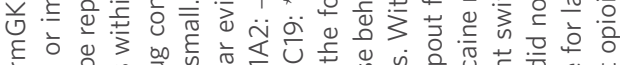

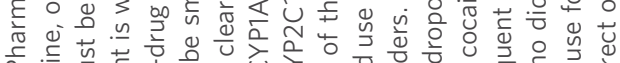

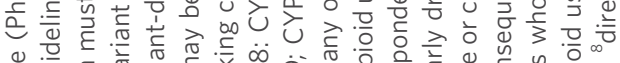

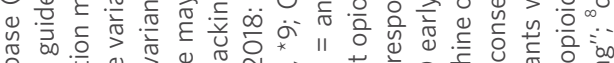

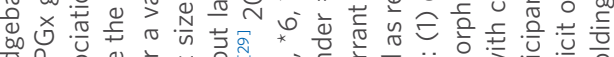
等

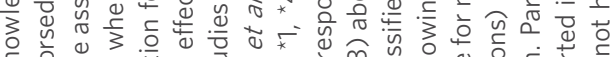

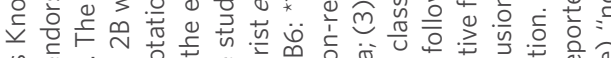

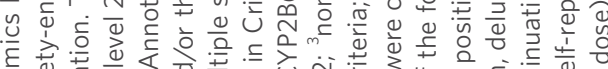

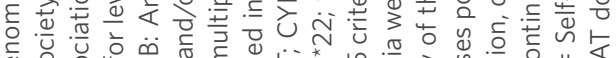

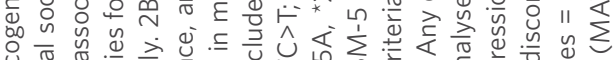

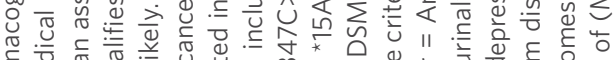

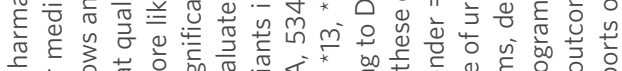

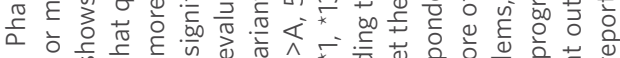

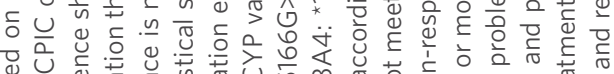

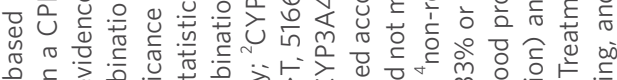

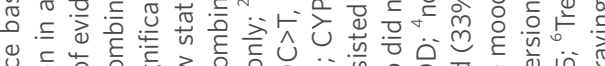

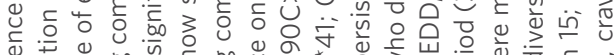

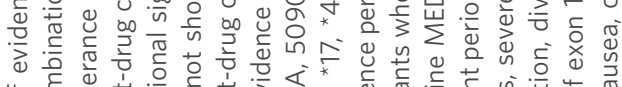

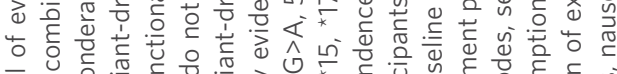

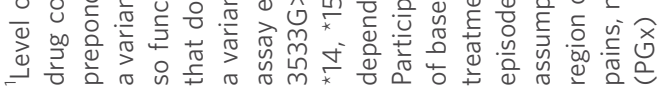


genetic factors. Lastly, as has been the case with most genetic research, greater diversity is needed in this field to ensure that the clinical gains from this research are broadly beneficial to all patients. Nevertheless, the development of PGx-guided strategies has the potential to reduce opioid misuse, improve clinical outcomes, and save healthcare resources.

\section{DECLARATIONS}

\section{Authors' contributions}

Participated in the initial conception of the manuscript: Arroyo Seguí H, Melin K, Santiago Quiñones D, Duconge J

Performed the majority of literature review and first draft of the manuscript: Arroyo Seguí $\mathrm{H}$, Melin K Provided critical review of the paper and editing of the final manuscript: Santiago Quiñones D, Duconge J Reviewed and approved the final manuscript for publication: Arroyo Seguí H, Melin K, Santiago Quiñones D, Duconge J

\section{Availability of data and materials}

Not applicable.

\section{Financial support and sponsorship}

Dr. Melin and Dr. Santiago Quiñones' work was partially supported by The National Institute of Health (NIH) (HCTRECD R25MD007607, HiREC S21MD001830) from the National Institute on Minority Health and Health Disparities; Dr. Melin's work was also partially supported by NIH (SC2DA052099) from the National Institute on Drug Abuse.

\section{Conflicts of interest}

All authors declared that there are no conflicts of interest.

\section{Ethical approval and consent to participate}

Not applicable

\section{Consent for publication}

Not applicable.

\section{Copyright}

(c) The Author(s) 2020.

\section{REFERENCES}

1. Center for Behavioral Health Statistics and Quality. 2015 National Survey on Drug Use and Health: Detailed Tables. Substance Abuse and Mental Health Services Administration; 2016. Available from: https:/www.samhsa.gov/data/sites/default/files/NSDUH-DetTabs-2015/ NSDUH-DetTabs-2015/NSDUH-DetTabs-2015.pdf. [Last accessed on 20 Jul 2020]

2. Schuckit MA. Treatment of Opioid-Use Disorders. N Engl J Med 2016;375:357-68.

3. Centers for Disease Control and Prevention. Understanding the Epidemic. Opioid Overdose. Available from: https://www.cdc.gov/ drugoverdose/epidemic/index.html. [Last accessed on 14 Jul 2020]

4. National Academies of Sciences, Engineering, and Medicine. Medications for opioid use disorder save lives. National Academies Press 2019.

5. Florence CS, Zhou C, Luo F, Xu L. The Economic Burden of Prescription Opioid Overdose, Abuse, and Dependence in the United States, 2013. Med Care 2016;54:901-6.

6. Jiang R, Lee I, Lee TA, Pickard AS. The societal cost of heroin use disorder in the United States. PLoS One 2017;12:e0177323.

7. First MB. DSM-5 handbook of differential diagnosis. American Psychiatric Pub; 2013.

8. Woody GE, Poole SA, Subramaniam G, Dugosh K, Bogenschutz M, et al. Extended vs short-term buprenorphine-naloxone for treatment of opioid-addicted youth: a randomized trial. JAMA 2008;300:2003-11.

9. Matson SC, Hobson G, Abdel-Rasoul M, Bonny AE. A retrospective study of retention of opioid-dependent adolescents and young adults 
in an outpatient buprenorphine/naloxone clinic. J Addict Med 2014;8:176-82.

10. Hadland SE, Bagley SM, Rodean J, Silverstein M, Levy S, et al. Receipt of timely addiction treatment and association of early medication treatment with retention in care among youths with opioid use disorder. JAMA Pediatr 2018;172:1029-37.

11. Clay SW. Treatment of addiction in the elderly. Aging health 2010;6:177-89.

12. Kotelchuck M, Cheng ER, Belanoff C, Cabral HJ, Babakhanlou-Chase H, et al. The prevalence and impact of substance use disorder and treatment on maternal obstetric experiences and birth outcomes among singleton deliveries in massachusetts. Matern Child Health J 2017;21:893-902.

13. Kaltenbach K, Finnegan LP. Developmental outcome of children born to methadone maintained women: a review of longitudinal studies. Neurobehav Toxicol Teratol 1984;6:271-5.

14. Jones HE, Kaltenbach K, Heil SH, Stine SM, Coyle MG, et al. Neonatal abstinence syndrome after methadone or buprenorphine exposure. N Engl J Med 2010;363:2320-31.

15. Schwartz RP, Gryczynski J, O'Grady KE, Sharfstein JM, Warren G, et al. Opioid agonist treatments and heroin overdose deaths in Baltimore, Maryland, 1995-2009. Am J Public Health 2013;103:917-22.

16. Mattick RP, Breen C, Kimber J, Davoli M, Breen R, et al. Methadone maintenance therapy versus no opioid replacement therapy for opioid dependence. Cochrane Database of Systematic Reviews. Chichester: John Wiley \& Sons, Ltd; 1996.

17. Kakko J, Svanborg KD, Kreek MJ, Heilig M. 1-year retention and social function after buprenorphine-assisted relapse prevention treatment for heroin dependence in Sweden: a randomised, placebo-controlled trial. Lancet 2003;361:662-8.

18. Bart G. Maintenance medication for opiate addiction: the foundation of recovery. J Addict Dis 2012;31:207-25.

19. Gowing L, Farrell MF, Bornemann R, Sullivan LE, Ali R. Oral substitution treatment of injecting opioid users for prevention of HIV infection. Cochrane Database Syst Rev 2011;10:CD004145.

20. Nicholls L, Bragaw L, Ruetsch C. Opioid dependence treatment and guidelines. J Manag Care Pharm 2010;16:S14-21.

21. Alderks CE. Trends in the use of methadone, buprenorphine, and extended-release naltrexone at substance abuse treatment facilities: 2003-2015 (Update). The CBHSQ report. Rockville, US: Substance Abuse and Mental Health Services Administration; 2017.

22. Potter JS, Dreifuss JA, Marino EN, Provost SE, Dodd DR, et al. The multi-site prescription opioid addiction treatment study: 18-month outcomes. J Subst Abuse Treat 2015;48:62-9.

23. Weiss RD, Potter JS, Griffin ML, Provost SE, Fitzmaurice GM, et al. Long-term outcomes from the national drug abuse treatment clinical trials network prescription opioid addiction treatment study. Drug Alcohol Depend 2015;150:112-9.

24. Jones HE, Fitzgerald H, Johnson RE. Males and females differ in response to opioid agonist medications. Am J Addict 2005;14:223-33.

25. McCance-Katz EF, Sullivan LE, Nallani S. Drug interactions of clinical importance among the opioids, methadone and buprenorphine, and other frequently prescribed medications: a review. Am J Addict 2010;19:4-16.

26. Litz M, Leslie D. The impact of mental health comorbidities on adherence to buprenorphine: A claims based analysis. Am J Addict 2017;26:859-63.

27. Lo A, Kerr T, Hayashi K, Milloy MJ, Nosova E, et al. Factors associated with methadone maintenance therapy discontinuation among people who inject drugs. J Subst Abuse Treat 2018;94:41-6.

28. Lopian KM, Chebolu E, Kulak JA, Kahn LS, Blondell RD. A retrospective analysis of treatment and retention outcomes of pregnant and/ or parenting women with opioid use disorder. J Subst Abuse Treat 2019;97:1-6.

29. Crist RC, Li J, Doyle GA, Gilbert A, Dechairo BM, et al. Pharmacogenetic analysis of opioid dependence treatment dose and dropout rate. Am J Drug Alcohol Abuse 2018;44:431-40.

30. Crist RC, Doyle GA, Nelson EC, Degenhardt L, Martin NG, et al. A polymorphism in the OPRM1 3'-untranslated region is associated with methadone efficacy in treating opioid dependence. Pharmacogenomics J 2018;18:173-9.

31. Crist RC, Clarke TK, Ang A, Ambrose-Lanci LM, Lohoff FW, et al. An intronic variant in OPRD1 predicts treatment outcome for opioid dependence in African-Americans. Neuropsychopharmacology 2013;38:2003-10.

32. Crettol S, Déglon JJ, Besson J, Croquette-Krokar M, Hämmig R, et al. ABCB1 and cytochrome P450 genotypes and phenotypes: influence on methadone plasma levels and response to treatment. Clin Pharmacol Ther 2006;80:668-81.

33. Csajka C, Crettol S, Guidi M, Eap CB. Population genetic-based pharmacokinetic modeling of methadone and its relationship with the QTc interval in opioid-dependent patients. Clin Pharmacokinet 2016;55:1521-33.

34. Levran O, Peles E, Hamon S, Randesi M, Adelson M, et al. CYP2B6 SNPs are associated with methadone dose required for effective treatment of opioid addiction. Addict Biol 2013;18:709-16.

35. Peng S, Jiang H, Du J, Lin S, Pan S, et al. Methadone dosage and plasma levels, SNPs of OPRM1 gene and age of first drug use were associated with outcomes of methadone maintenance treatment. Front Genet 2018;9:450.

36. Tian JN, Ho IK, Tsou HH, Fang CP, Hsiao CF, et al. UGT2B7 genetic polymorphisms are associated with the withdrawal symptoms in methadone maintenance patients. Pharmacogenomics 2012;13:879-88.

37. Berrettini W. A brief review of the genetics and pharmacogenetics of opioid use disorders. Dialogues Clin Neurosci 2017;19:229-36.

38. Crist RC, Clarke TK, Berrettini WH. Pharmacogenetics of opioid use disorder treatment. CNS Drugs 2018;32:305-20.

39. Gerra G, Borella F, Zaimovic A, Moi G, Bussandri M, et al. Buprenorphine versus methadone for opioid dependence: predictor variables for treatment outcome. Drug Alcohol Depend 2004;75:37-45.

40. Serafini G, Adavastro G, Canepa G, De Berardis D, Valchera A, et al. The efficacy of buprenorphine in major depression, treatmentresistant depression and suicidal behavior: a systematic review. Int J Mol Sci 2018;19:2410.

41. Toce MS, Chai PR, Burns MM, Boyer EW. Pharmacologic treatment of opioid use disorder: a review of pharmacotherapy, adjuncts, and toxicity. J Med Toxicol 2018;14:306-22. 
42. Zhang H, Kranzler HR, Yang BZ, Luo X, Gelernter J. The OPRD1 and OPRK1 loci in alcohol or drug dependence: OPRD1 variation modulates substance dependence risk. Mol Psychiatry 2008;13:531-43.

43. Beer B, Erb R, Pavlic M, Ulmer H, Giacomuzzi S, et al. Association of polymorphisms in pharmacogenetic candidate genes (OPRD1, GAL, ABCB1, OPRM1) with opioid dependence in European population: a case-control study. PLoS One 2013;8:e75359.

44. Saxon AJ, Ling W, Hillhouse M, Thomas C, Hasson A, et al. Buprenorphine/Naloxone and methadone effects on laboratory indices of liver health: a randomized trial. Drug Alcohol Depend 2013;128:71-6.

45. Blanco F, Muriel C, Labrador J, Gonzalez-Porras JR, Gonzalez-Sarmiento R, et al. Influence of UGT2B7, CYP3A4, and OPRM1 gene polymorphisms on transdermal buprenorphine pain control in patients with critical lower limb ischemia awaiting revascularization. Pain Pract 2016;16:842-9.

46. Muriel J, Margarit C, Planelles B, Serralta MJ, Puga C, et al. OPRM1 influence on and effectiveness of an individualized treatment plan for prescription opioid use disorder patients. Ann N Y Acad Sci 2018;1425:82-93.

47. Nelson EC, Lynskey MT, Heath AC, Wray N, Agrawal A, et al. Association of OPRD1 polymorphisms with heroin dependence in a large case-control series. Addict Biol 2014;19:111-21.

48. Crist RC, Phillips KA, Furnari MA, Moran LM, Doyle GA, et al. Replication of the pharmacogenetic effect of rs678849 on buprenorphine efficacy in African-Americans with opioid use disorder. Pharmacogenomics J 2019;19:260-8.

49. Clarke TK, Crist RC, Ang A, Ambrose-Lanci LM, Lohoff FW, et al. Genetic variation in OPRD1 and the response to treatment for opioid dependence with buprenorphine in European-American females. Pharmacogenomics J 2014;14:303-8.

50. Moody DE, Fang WB, Morrison J, McCance-Katz E. Gender differences in pharmacokinetics of maintenance dosed buprenorphine. Drug Alcohol Depend 2011;118:479-83.

51. Zubieta JK, Dannals RF, Frost JJ. Gender and age influences on human brain mu-opioid receptor binding measured by PET. Am J Psychiatry 1999;156:842-8.

52. Unger A, Jung E, Winklbaur B, Fischer G. Gender issues in the pharmacotherapy of opioid-addicted women: buprenorphine. J Addict Dis 2010;29:217-30.

53. Nunes EV, Levin FR. Treatment of depression in patients with alcohol or other drug dependence: a meta-analysis. JAMA 2004;291:1887-96.

54. Brewer DD, Catalano RF, Haggerty K, Gainey RR, Fleming CB. A meta-analysis of predictors of continued drug use during and after treatment for opiate addiction. Addiction 1998;93:73-92.

55. Gerra G, Somaini L, Leonardi C, Cortese E, Maremmani I, et al. Association between gene variants and response to buprenorphine maintenance treatment. Psychiatry Res 2014;215:202-7.

56. Russo SJ, Nestler EJ. The brain reward circuitry in mood disorders. Nat Rev Neurosci 2013;14:609-25.

57. Wee S, Koob GF. The role of the dynorphin-kappa opioid system in the reinforcing effects of drugs of abuse. Psychopharmacology 2010;210:121-35.

58. Blum K, Sheridan PJ, Wood RC, Braverman ER, Chen TJ, et al. The D2 dopamine receptor gene as a determinant of reward deficiency syndrome. J R Soc Med 1996;89:396-400.

59. Lawford BR, Young RMD, Noble EP, Sargent J, Rowell J, et al. The D2 dopamine receptor A1 allele and opioid dependence: association with heroin use and response to methadone treatment. Am J Med Genet 2000;96:592-8.

60. Barratt DT, Coller JK, Somogyi AA. Association between the DRD2 A1 allele and response to methadone and buprenorphine maintenance treatments. Am J Med Genet B Neuropsychiatr Genet 2006;141:323-31.

61. Picard N, Cresteil T, Djebli N, Marquet P. In vitro metabolism study of buprenorphine: evidence for new metabolic pathways. Drug Metab Dispos 2005;33:689-95.

62. Cone EJ, Gorodetzky CW, Yousefnejad D, Buchwald WF, Johnson RE. The metabolism and excretion of buprenorphine in humans. Drug Metab Dispos 1984;12:577-81.

63. Zanger UM, Schwab M. Cytochrome P450 enzymes in drug metabolism: regulation of gene expression, enzyme activities, and impact of genetic variation. Pharmacol Ther 2013;138:103-41.

64. Rouguieg K, Picard N, Sauvage FL, Gaulier JM, Marquet P. Contribution of the different UDP-glucuronosyltransferase (UGT) isoforms to buprenorphine and norbuprenorphine metabolism and relationship with the main UGT polymorphisms in a bank of human liver microsomes. Drug Metab Dispos 2010;38:40-5.

65. Ettienne EB, Chapman E, Maneno M, Ofoegbu A, Wilson B, et al. Pharmacogenomics-guided policy in opioid use disorder (OUD) management: an ethnically-diverse case-based approach. Addict Behav Rep 2017;6:8-14.

66. Westlind-Johnsson A, Hermann R, Huennemeyer A, Hauns B, Lahu G, et al. Identification and characterization of CYP3A4*20, a novel rare CYP3A4 allele without functional activity. Clin Pharmacol Ther 2006;79:339-49.

67. Ettienne EB, Ofoegbu A, Maneno MK, Briggs J, Ezeude G, et al. Pharmacogenomics and opioid use disorder: clinical decision support in an African American cohort. J Natl Med Assoc 2019;111:674-81.

68. Muriel J, Margarit C, Barrachina J, Ballester P, Flor A, et al. Pharmacogenetics and prediction of adverse events in prescription opioid use disorder patients. Basic Clin Pharmacol Toxicol 2019;124:439-48.

69. Whirl-Carrillo M, McDonagh EM, Hebert JM, Gong L, Sangkuhl K, et al. Pharmacogenomics knowledge for personalized medicine. Clin Pharmacol Ther 2012;92:414-7. 